\title{
Research on satellite communication network based on PCC architecture
}

\author{
Yu Xiao ${ }^{1,2}$, Zhao Jinfeng ${ }^{2,}$, , Wei Lai ${ }^{2}$, Guo Ruiyu ${ }^{2}$ \\ ${ }^{1}$ College of Information Science and Engineering, Northeastern University, China; \\ ${ }^{2}$ Communication Engineering Design and Research Institute of PLAs, China \\ a13feng@sina.com
}

Keyword: Satellite Communication; PCC; PCRF; QoS

\begin{abstract}
In the conventional satellite communication networking process, face with many problems, such as resource utilization is not high, QoS is weak, lack of real-time dynamic adjustment to link. PCC architecture-based satellite communications network model through a secure channel to receive channel real-time monitoring information, enhance the satellite resources utilization and QoS based on the policy mechanism, and improve the user experience. On the basis of given networking process, the function units is described in detail, which lays the foundation for the next step of the simulation.
\end{abstract}

\section{Introduction}

With the development of satellite communications, the duration and frequency of voice services and IP services are growths rapidly, to the original is not abundant satellite resources brought great pressure. Due to the limitation of satellite resources, a lot of problems have emerged, such as the service quality has been reduced, service reliability and availability have been reduced, and the user experience has become worse. How to ensure the voice, streaming media and other services under the existing circumstances of the quality of service becomes an issue. In $3 \mathrm{G}$ or LTE mobile communication network, commonly used Policy and Charging Control (PCC) to solve the problem caused by the surge in network data traffic ${ }^{[1]}$, improve bandwidth resource utilization and QoS. At present, the PCC architecture has played a good use efficiency, which has brought enormous economic benefits for mobile operators. The development of satellite communication network is slightly lagging behind the terrestrial mobile communications network, the PCC architecture is used into the satellite communication network which can effectively solve the problem of restricting the development of resources, and can promote the seamless connection between the satellite network and ground network.

\section{PCC}

R7 3GPP proposed PCC architecture, based on the packet domain to achieve service policy control. R8 on the basis of R7 increased access to LTE, CDMA, WLAN, and other access types, enhanced roaming and PCRF addressing function. On the basis of the R8, R9 further improve the structure and other parts. The concepts of policy level is proposed by R10, and carry out the enhancement function research, such as service inspection report, SPR standards, based on the service network selection ${ }^{[2]}$, etc.. PCC architecture is mainly composed by PCRF, PCEF, SPR, AF and other functional entities, the standard architecture is shown in the following diagram ${ }^{[3][4]}$.

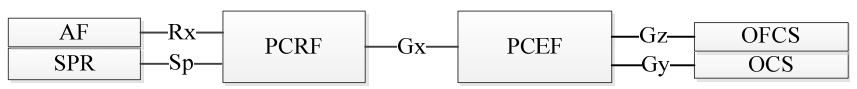

Figure 1 PCC architecture diagram

AF, Application Function, according to the service protocol with the user, the application layer session information is dynamically provided to PCRF.

SPR, Subscription Profile Repository, the contract based policy and the user's information are 
provided. SPR contract information includes: user class, user level, subscription the package of information, the user's QoS information, etc.

PCRF, Policy and Charging Rule Function, this functional entity contains the policy control decision and flow based charging control functions. PCRF accepts input from the PCEF, SPR and the $\mathrm{AF}$, at the same time, considering the real-time information of the network, the user's subscription information, system time and other information, integrated above information for policy decision, and sent the decision results to the PCEF, real-time dynamic adjustment user mobile broadband policy .

PCEF, Policy and Charging Enforcement Function, mainly includes detection and policy execution of service data flow and flow based charging function.

OCS, Online Charging System, responsible for the collection of each network element billing event to generate billing data records, OCS can trigger PCRF initiated IP-CAN bearer services at any time.

OFCS, Offline Charging System, can affect the charging mechanism in real time.

Rx: between AF and PCRF, for transmitting application layer information from AF to PCRF, including differentiated billing information and for media application bandwidth requirements of QoS control, etc.

Sp: between SPR and PCRF, for PCRF obtain IP - CAN transfer policy related to the user information from the SPR, such as user ID, PDN identification, etc.

Gx: between PCEF and PCRF, for delivering policy and charging rules. The interface supports PCC information transmission of SDF (Data Flow Service) level, and supports the transmission of wireless access technology information and location information.

Gy: between PCEF and OCS, for transmitting the online billing control information.

Gz: between PCEF and OFCS, for transmitting the data stream based on offline charging.

\section{Satellite Communication Network}

\subsection{Traditional satellite communication networking mode}

At present, the network of satellite communication needs three links, which are resource application, decision support and resource allocation ${ }^{[5]}$, as shown in the following figure. The resource application means apply satellite resources required for communication, according to the location of the two sides, service type, occupancy time and other information; Network control center station receive the resource application, then make resource planning and evaluation, according to the whole network service usage and utilization of satellite resources, propose an optimized decision support plan; In the resource allocation, the resource allocation instruction is launched to the satellite, then the communication users can use the satellite resources in the application period.

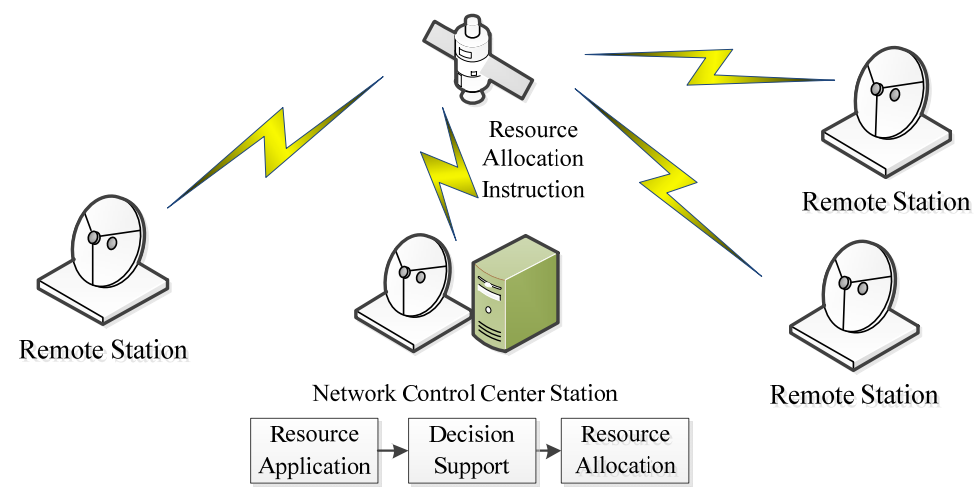

Figure 2 Sketch map of traditional satellite communication network

There are the following problems in the above network process:

(1) In resource application, applicants need to be aware of the basic information of the antenna diameter, geographical location and other basic information of all users within the network, in reality it's very difficult, applicants can only grasp the local information, so there is a large amount 
of redundancy in the resource application of satellite beam, bandwidth and power.

(2) In decision support, there is no user priority QoS, nor the priority of different users to initiate different service QoS. For example, when a low level user initiates a voice service, it is unable to perform a high priority QoS only based on the voice service standard.

(3) The resource allocation instructions of the network control center station adopt one launch mode, and then after the end of the application period, the instruction to recover the resources is launched again. Although this model reduces the occupancy of satellite resources, but with the cost of losing flexibility. When the communication service type change, the network control center station can't be adjusted in time, need to experience the links: resource application, decision support, resource allocation, the user experience is poor.

(4) In the process of the whole network, users, service and decision is a sequential relationship, not a good overall planning; In addition, as there is no established link between the network control center and the satellite, therefore, the channel quality monitoring information can't be received. When the quality of the channel deterioration, unable to make timely adjustments to ensure that the communication resources indicators.

\subsection{PCC architecture-based satellite communications networking mode}

PCC satellite communications network mode requires network control center station and the satellite to establish a long-term security channel, which responsible for allocating resources, QoS and dynamically adjusting link resources according to channel quality and service change, users only need to concern about with who initiated what kind of service.

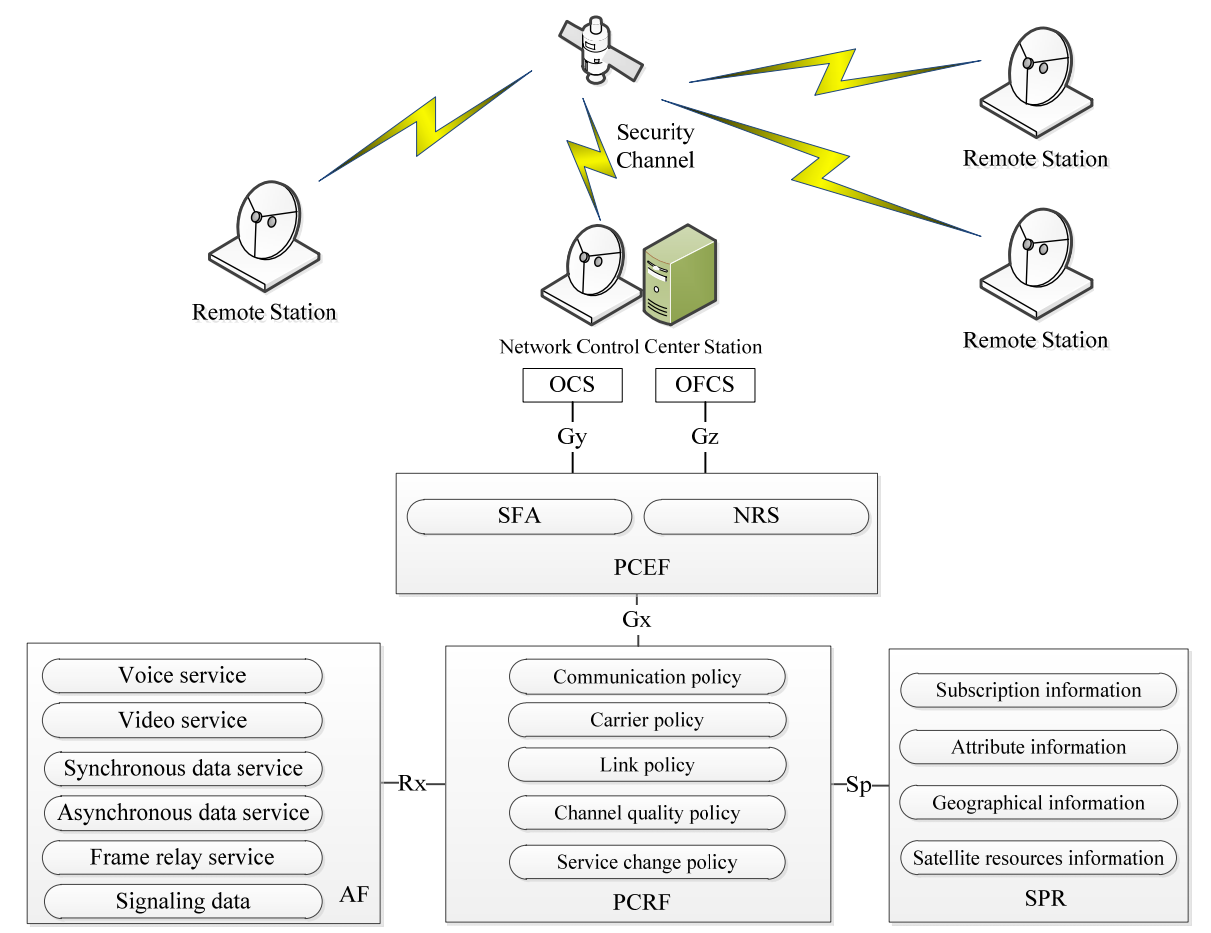

Figure 3 Sketch map of PCC satellite communication network

SPR. In the 3GPP PCC architecture SPR only store user related information, but in the satellite communication network, satellites as storage and forwarding node, the resource information has an important influence on the strategic planning. So, the information stored in SPR mainly includes the subscription information, the attribute information, the geographic information and the satellite resources information:

Subscription information refers to usage period, usage frequency, history record and the maximum limit of the user.

Attribute information includes station type information, antenna information, and identifier information. Station type information refers to fixed station and mobile station. The fixed station is divided into the central station, the regional station, the remote station and the monitoring station; The mobile station is divided into vehicle, ship, airborne and portable. Antenna information 
includes antenna aperture, antenna gain and power amplifier. Identifier information means that each user has a unique global identifier that is used to distinguish the user's priority.

Geographical information refers to the user's coordinate position, weather information and the ionosphere, troposphere and other spatial information.

Satellite resources information includes satellite orbital position, beam radius, transponder bandwidth, frequency, amplifiers and other resources information.

AF. AF can provide voice service, video service, synchronous data service, asynchronous data service, frame relay service and signaling data, etc. Each service requires different requirements on network delay, channel bandwidth, so divide different QCI levels, as shown in the following table:

Table 1 QCI and priority class table

\begin{tabular}{|l|l|l|l|}
\hline Service name & QCI & Priority & Delay budget \\
\hline Voice service & 1 & 2 & $150 \mathrm{~ms}$ \\
\hline Video service & 2 & 3 & $200 \mathrm{~ms}$ \\
\hline Synchronous data service & 3 & 4 & $200 \mathrm{~ms}$ \\
\hline Asynchronous data service & 4 & 5 & $300 \mathrm{~ms}$ \\
\hline Frame relay service & 5 & 6 & $250 \mathrm{~ms}$ \\
\hline Signaling data & 6 & 1 & $150 \mathrm{~ms}$ \\
\hline
\end{tabular}

PCRF. PCRF includes communication policy, carrier policy, link policy, channel quality policy and service change policy.

Communication policy, firstly, according network users of geographic information, attribute information and satellite resources information, which stored in SPR, to calculate the cover beam, the beam type can be divided into spot beams, global beam, and land beam, etc. General user uses land beam and global beam, high level user can use spot beam. Secondly, under the beam choose transparent transponder or processing transponder, when the users need multiple beams or high level users, need to use processing transponder, which can improve the transmission qualities of satellite signals.

Carrier policy based on the SPR user attributes information and AF service level using different coding methods, modulation methods, carrier quantity and bandwidth. At the same time, in the communication process to receive the channel quality policy instruction, dynamically adjusting coding methods and modulation methods.

Link policy refers to the link budget and decision optimization according the communication policy and carrier policy, and set the system margin such as rainfall margin, interrupt margin, demodulation margin, propagation margin. The rainfall margin is determined by the weather condition and the performance of the receiving system; The interrupt margin is determined by the share of the RF noise in the system; The demodulation margin is determined by the performance of the modulator methods and demodulator; The propagation margin is determined by the use of the coding method and the interference situation.

Channel quality policy refers to real-time monitoring channel quality among network users through the long-term security channel, return the channel quality indicator (CQI) ${ }^{[6]}$, CQI is determined by the SNR of the uplink and downlink signals, support periodic and non-periodic reporting. High CQI value indicates good quality of the channel, according different CQI value choose different coding methods and modulation methods.

Service change policy refers to real-time monitoring service usage among network users through the long-term security channel, respond the service change application (SCA), according to the user attribute information and the utilization of the whole network resources, give the answer of rejection, delayed approval and immediate approval, and feedback to the application users.

PCEF、SFA、OCS and OFCS. PCEF is composed by service flow authorization (SFA) and network running state (NRS), mainly complete policy implementation and feedback information collection. Firstly, use the control antenna to launch the operation instructions through the long-term security channel, reserve and distribute satellite resources according the policy planning, 
initiate and establish various types of application services; Secondly, PCEF is responsible collecting the user's service usage information and satellite resources occupation information, report to PCRF through the Gx interface periodic and non-periodic; Finally, PCEF is responsible for monitoring the user and generate billing records, transfer to OCS and OFCS through the Gy and Gz interface, OCS and OFCS generate billing results based on billing mechanisms.

\section{PCC architecture-based satellite communications networking process}

Figure 4 shows a process of PCC architecture-based satellite communications networking, the following example shows the implementation of the signaling process by using the remote station as an example.

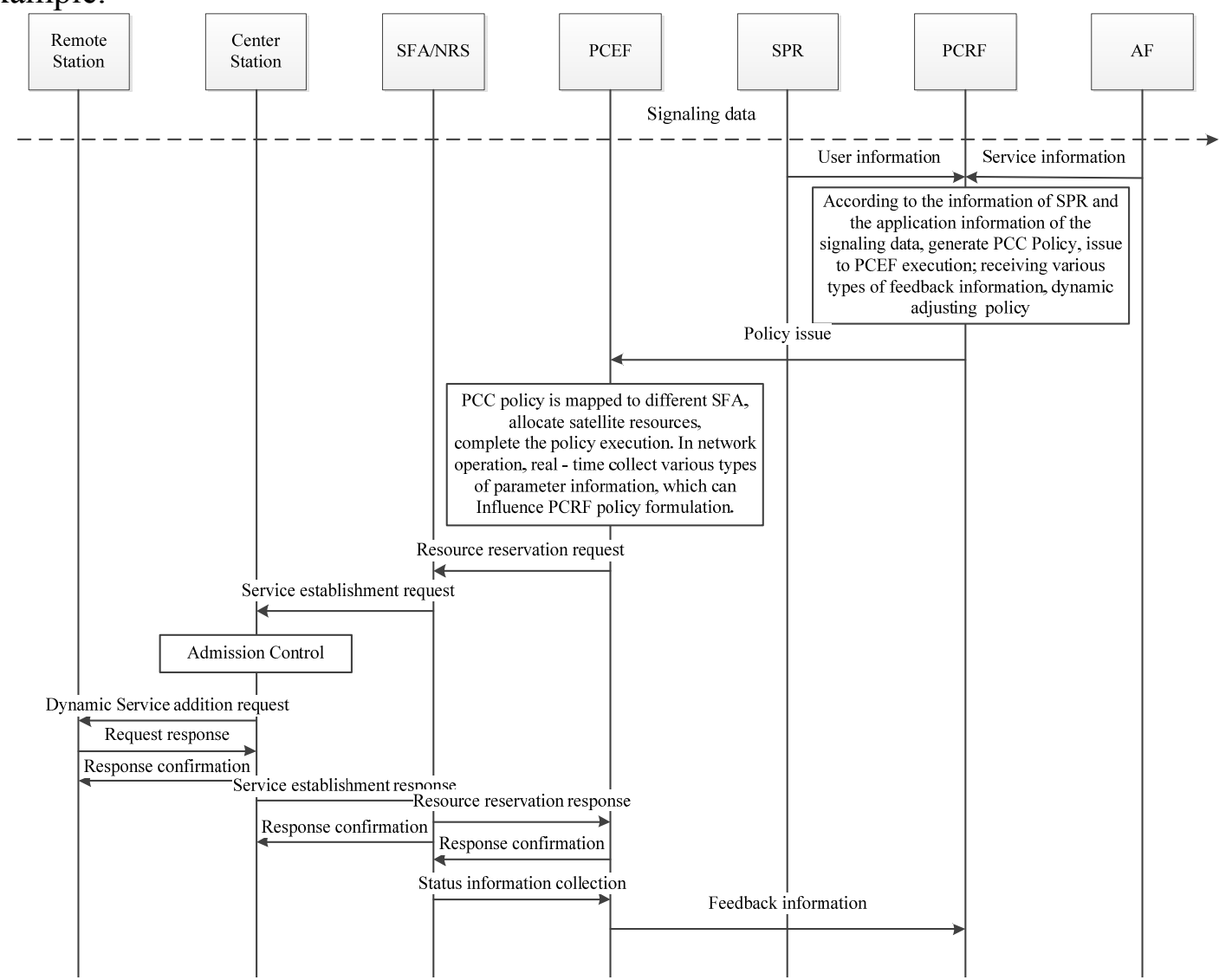

Figure 4 PCC satellite communications networking process

(1) Remote station initiate service, SPR provide remote station related information to PCRF by the $\mathrm{Sp}$ interface, AF provide service information to PCRF by the Rx interface, PCRF according to SPR and AF to formulate the policy, through the Gx interface issue the policy to PCEF.

(2) PCEF receives PCRF policy, complete the local mapping of the policy and bind the corresponding execution module. SFA is responsible for the service flow authorization, reserve and distribute the satellite resources, sent the service establishment request to the center station network control system.

(3) The center station network control system sent dynamic service addition request to the remote station according service establishment request, and it is also responsible for the admission control of the remote station.

(4) Remote stations respond to dynamic service addition request, according to the situation of the station to give the answer of access, give up or delay access, then sent the message to the network control system.

(5) The center station network control system receives request response, and sent response confirmation. At the same time, sent service establishment response to SFA, then SFA return response confirmation.

(6) During the service normally running, NRS sent Resource reservation response to PCEF, and 
then PCEF return response confirmation. NRS start collecting network running status information, PCEF integrated network status and the policy implementation, sent the feedback information to PCRF. PCRF receive feedback information, and evaluate the effectiveness of policy implementation, optimization of policy decisions and stored in the policy database to provide a basis for the next policy making.

\section{Summary}

Satellite communication is an important means to solve the global network coverage in the future, with good prospects for development ${ }^{[7]}$. PCC architecture is an effective way to improve the quality of network service, this paper makes a preliminary exploration on the application of PCC architecture in the satellite communication network, the system framework is built, and the related functional units are described in detail. The next step will be simulate the performance of PCC architecture-based satellite communications networking, calculate the quantitative assessment conclusions, and tap the potential advantages.

\section{Reference}

[1] ZHANG Mei-jing, WANG Ying-ming, LI Ling-hui. Research on control policy of data service based on PCC architecture. Journal of Fuzhou University (Natural Science Edition) Vol.42 No.2, (2014), p23-241.

[2] Gu Ming, PCC Architecture Applicated in LTE Network, TELECOM ENGINEERING TECHNICS AND STANDARDIZATION, No.16, (2010), p35-39.

[3] LIU Wei, GUO Jia-qi, WANG Bin, Research on PCC control strategy mechanism and deployment in network. TELECOM ENGINEERING TECHNICS AND STANDARDIZATION, No.12, (2011), p48-52.

[4] $\mathrm{Xu}$ Gen, Research and design of policy and charging control in LTE networks, East China Normal University Master's Dissertation, 2013.

[5] HE Jian, WANG Zu-lin, ZHONG Xuan-ming, LI Xi. Policy Refinement of QoS Management in Satellite Communication System. Computer Engineering Vol.35 No.16 (2009) p7-9.

[6] 3GPP TS 36.213, V10.1.0, (2011).

[7] Yu Xiao, Zhao jinfeng, Feng jun, Lei Weimin Research on the Ku Band Rain Attenuation Estimation Algorithm, CICT, (2015), p583-586. 